\section{Basal cell carcinoma of the eyelid in children: a report of three cases}

A Al-Buloushi', ${ }^{1,}$,JPS Filho ${ }^{3,4}$, A Cassie $^{3}$,

B Arthurs ${ }^{1,2}$ and MN Burnier $\mathrm{Jr}^{3}$
Abstract

Basal cell carcinoma is the most common eyelid malignancy, accounting for approximately $90 \%$ of malignant eyelid tumours. Despite its prevalence in adults, it is extremely rare in children, and usually occurs in the setting of a known genetic defect or following radiotherapy treatment. We report three cases of de novo basal cell carcinoma in children. These children had no known genetic syndromes and had not undergone radiotherapy.

Eye (2005) 19, 1313-1314. doi:10.1038/sj.eye.6701758; published online 14 January 2005

Keywords: BCC; basal cell carcinoma; eyelid; children

Basal cell carcinoma (BCC) is the most common eyelid malignancy, accounting for approximately $90 \%$ of malignant eyelid tumours. These tumours are most commonly located on the lower eyelid margin and the medial canthus. ${ }^{1}$

BCC has a peak incidence in the seventh decade of life. ${ }^{1}$ Despite its prevalence in adults, it is extremely rare in children. ${ }^{2}$ When found in the paediatric age group, BCC is usually associated with a positive family history of particular syndromes and should therefore prompt inquiry into possible systemic associations such as basal cell nevus syndrome (Gorlin syndrome), xeroderma pigmentosum, and nevus sebaceous. ${ }^{2,3}$ The development of BCC in children may also occur following radiotherapy. ${ }^{3}$

We report three cases of de novo BCC in children. These children had no known genetic syndromes and had not undergone radiotherapy.

\section{Case reports}

Case 1

A 17-year-old Caucasian girl presented to a general ophthalmologist at the McGill University Health Centre in Montreal, Canada, with a $5 \mathrm{~mm}$ wide lesion of the left lower lid (LLL). The lesion was felt to be a nevus and the decision was made to observe the lesion and have the patient return for a follow-up visit in 6 months. At the time of follow-up the patient noted that the lesion had bled on one or two occasions. Examination of the lesion revealed small erosion in the centre, which made it suspicious for BCC (Figure 1). The patient was referred to the oculoplastic service.

The patient had no past medical history and there was no family history of skin cancer. However, she did admit to a history of more than one bad sunburn as a child.

Histopathologic assessment of a biopsied specimen confirmed that the lesion was a morpheaform (sclerosing) type of BCC. A full thickness excision of the lesion was performed 1 week later. The margins were analysed by frozen section technique and found to be free of neoplastic involvement. The lid was reconstructed using a Tenzel semicircular rotational flap technique.

\section{Case 2}

Case number 2 represents a consultation report of a 14-year-old Caucasian girl who presented with a nodular indurated lesion of the right lower lid of 10-months duration. Her mother reported that the lesion had grown over the last 4 months, particularly during the summer when the girl had worked as a lifeguard at a local swimming pool. The patient had no past medical history. The lesion was completely excised. Histopathologic examination showed the specimen to be a nodular (basaloid) type of
${ }^{1}$ Department of Ophthalmology, McGill University Health Center, Montreal General Hospital, 3775, University St, Room 216, Montreal, Canada

${ }^{2}$ Royal Victoria Hospital, Montreal, Canada

${ }^{3}$ Henry C. Witelson Ocular Pathology Laboratory, Department of Ophthalmology McGill University, Montreal, Canada

${ }^{4}$ Department of Ophthalmology, Federal University of São Paulo,

São Paulo, Brazil

Correspondence: B Arthurs, 1650 Cedar Avenue, Montreal General Hospital, Pavillom Livingnston, Room L4-116, Montreal, Quebec H3G 1A4, Canada Tel: 1514 9341934×48055; Fax: 15149348223.

E-mail: bryan.arthurs@ staff.mcgill.ca

Received: 15 July 2004 Accepted: 23 September 2004

Published online:

14 January 2005

Financial interest: None 


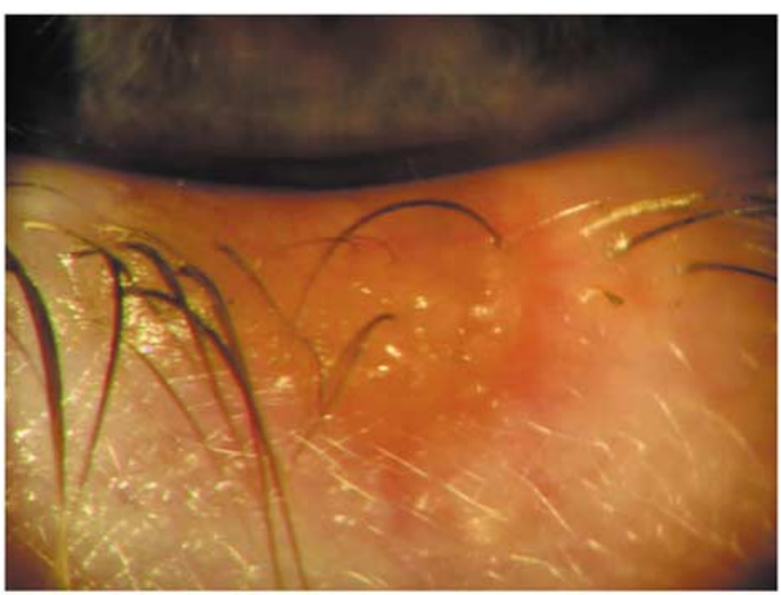

Figure 1 Slit-lamp examination of case 1 showing a $5 \mathrm{~mm}$-wide lesion of the left lower lid.

BCC. The surgical margins were free of neoplastic involvement.

\section{Case 3}

Case number 3 represents a consultation report of an 8year-old Hispanic boy who presented with a nodular mass of LLL. The patient's medical history was unremarkable. The lesion was completely excised. Histopathologic examination showed the $20 \times 12 \times 7 \mathrm{~mm}^{3}$ specimen to be a nodular (basaloid) type of BCC. The surgical margins were free of neoplastic involvement.

\section{Comment}

We are hereby reporting three cases of eyelid BCC in children with no history of a previous skin cancer, syndrome, or radiation treatment. This paper therefore presents a collection of very rare cases. The first case had previous bad sunburns, and the second had a history of sun exposure. In the third case, which was a consultation case, it was not possible to obtain information regarding sun exposure. BCC is more likely to occur in fair-skinned individuals ${ }^{1}$ and the length of actinic exposure plays a direct role in the frequency of tumour occurrence. However, the peak incidence of BCC occurs in the seventh decade of life, and in the paediatric age group, solitary BCC is rare and usually occurs in the setting of a known genetic defect or following radiotherapy treatment. ${ }^{2,3}$ Few papers have reported eyelid BCC in children without such medical history.

Keramidas and Anagnostou ${ }^{4}$ described the youngest reported patient with eyelid BCC, who was a 27-monthold infant with a BCC affecting the LLL in the absence of any predisposing cutaneous diseases. The lesion appeared 4 months before consultation and developed rapidly to become ulcerated. LeSueur $e t a l^{2}$ reported another case of BCC in the LLL of an 8-year-old boy, with a history of several severe sunburns. Rahbari and Mehregan $^{3}$ found that 85 out of 390000 dermatopathologic specimens were BCCs in patients 6-19 years old. Six of these 85 patients had eyelid BCC; however, it is not clear if any of these children had a known genetic defect or had received radiation treatment.

It is not clear if BCC is more aggressive in paediatric patients than in adults. In a retrospective review. Leffel et $a l^{5}$ found an increased occurrence of aggressive-growth BCC in patients younger than 35 years old compared with older patients. In our cases, the histopathology of the first case was a morpheaform (sclerosing) type. This type of BCC is particularly aggressive. ${ }^{1}$ In the second and third cases, the lesions were nodular (basaloid) types.

We recommend that paediatricians and general ophthalmologists suspect BCC in children or teenagers, who present with a nonhealing ulcer or pearly indurated eyelid lesion with telengiectatic vessels on the surface. This suspicion should be particularly high in those patients with clinical evidence of photodamage, such as freckling, or a history of previous sunburns or radiation therapy. Early detection will permit treatment of smaller tumours and minimize the ultimate cosmetic defect.

\section{References}

1 Font RL. Eyelid and lacrimal drainage system. In: Spencer WH (ed). Ophthalmic Pathology: An Atlas and Textbook, 4th edn. WB Saunders: Philadelphia, 1996, pp 2249-2257.

2 LeSueur BW, Silvis NG, Hansen RC. Basal cell carcinoma in children: report of 3 cases. Arch Dermatol 2000; 136(3): 370-372.

3 Rahbari H, Mehregan AH. Basal cell epithelioma (carcinoma) in children and teenagers. Cancer 1982; 49(2): 350-353.

4 Keramidas DC, Anagnostou D. Basal-cell carcinoma of the lower lid in a 27-month-old child. Z Kinderchir 1987; 42(4): 250-251.

5 Leffell DJ, Headington JT, Wong DS, Swanson NA. Aggressive-growth basal cell carcinoma in young adults. Arch Dermatol 1991; 127(11): 1663-1667. 